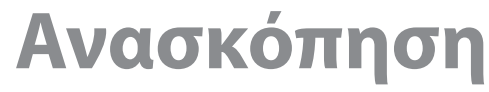

\section{To Hovomáti ths Kuvoupevívns

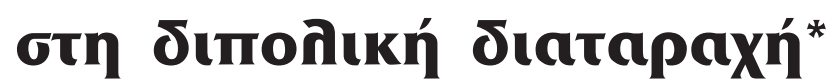

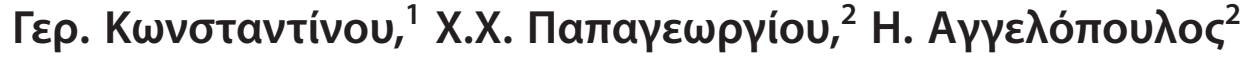

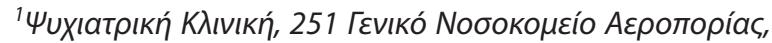

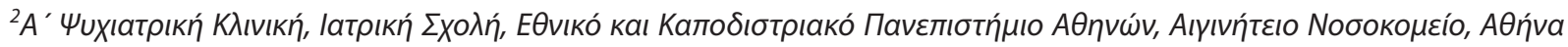

Uuxıатрıкń 2018, 29:338-348

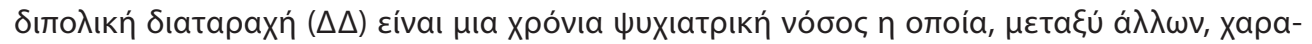

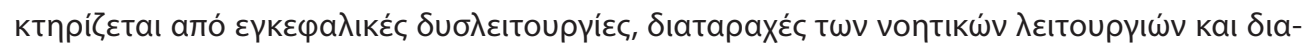

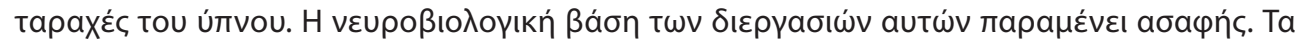

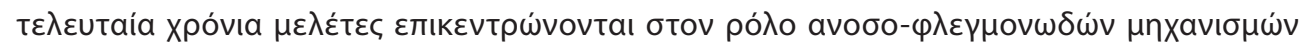

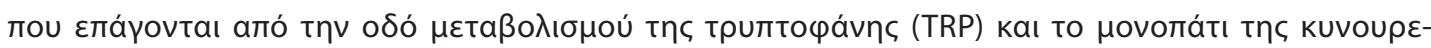

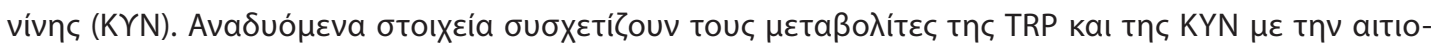

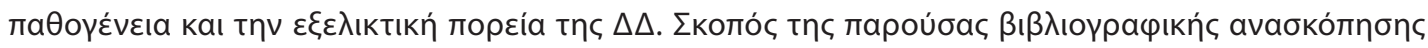

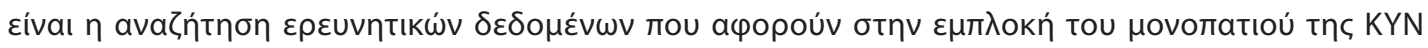

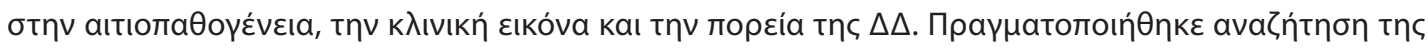

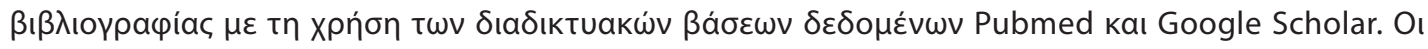

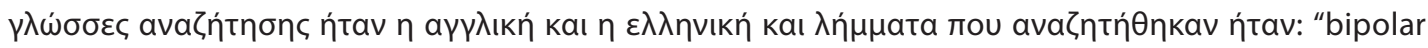
disorder", "depression", "mania", "tryptophan", "kynurenine pathway, "cognitive dysfunction", "sleep

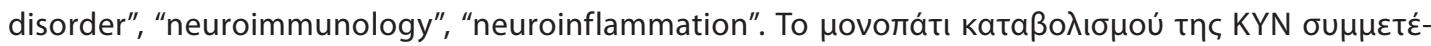

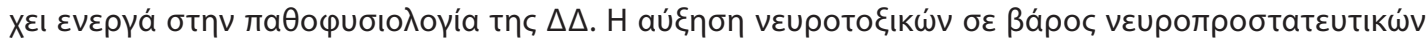

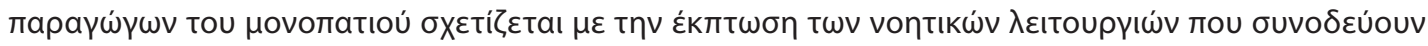

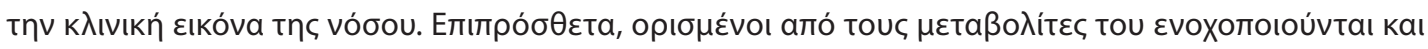

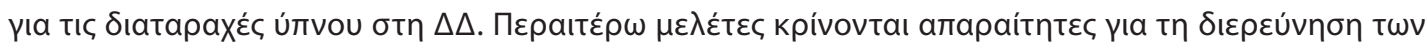

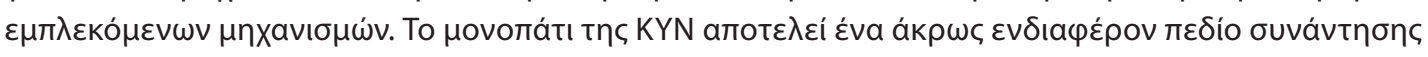

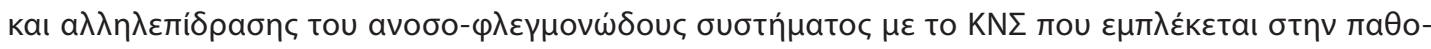

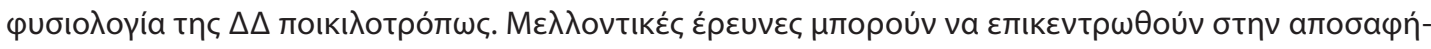

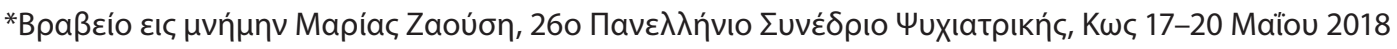




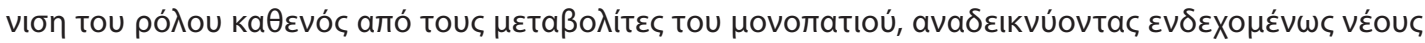

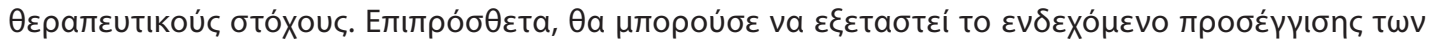

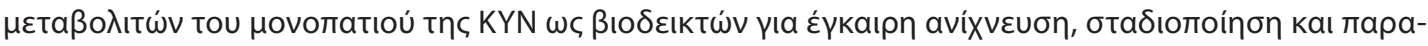

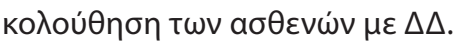

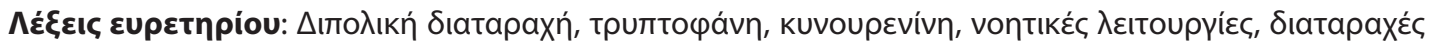

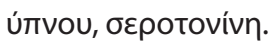

\section{Eıaaywyń}

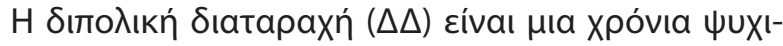

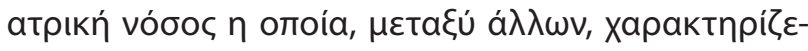

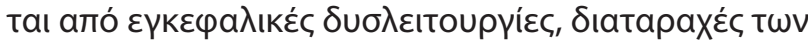

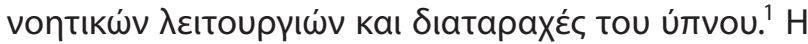

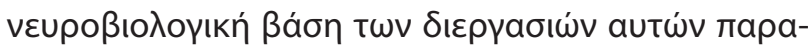

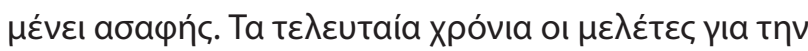

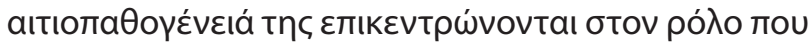

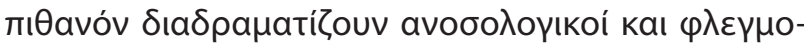

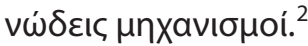

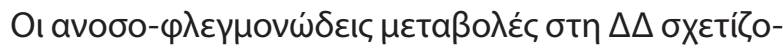

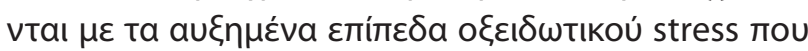

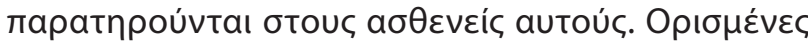

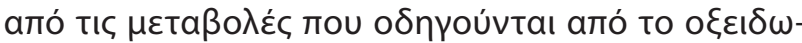

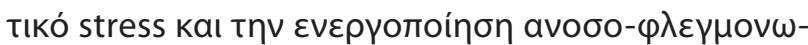

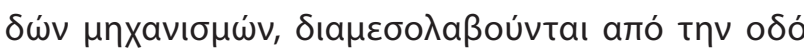

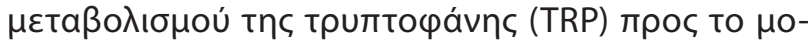

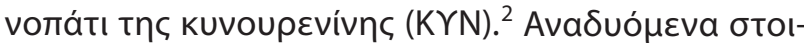

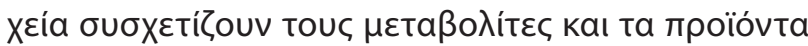

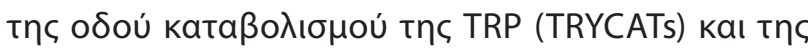

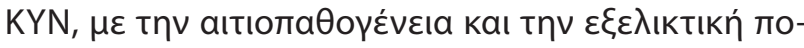
pzía tnc $\Delta \Delta$. .

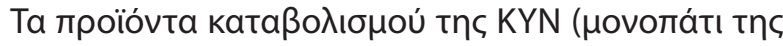

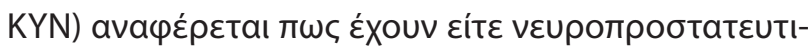

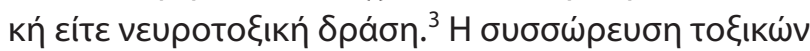

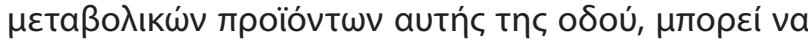

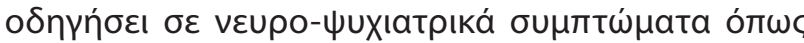

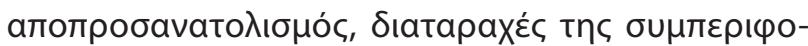

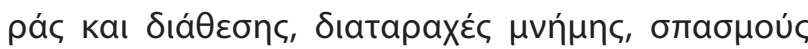

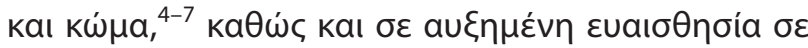

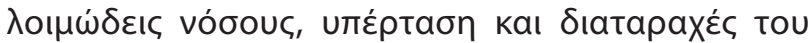

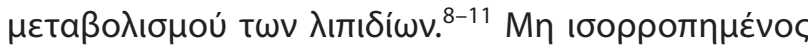

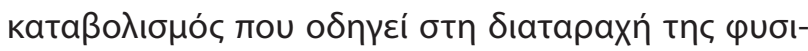

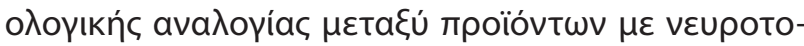

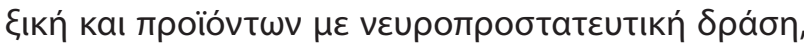

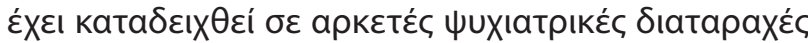

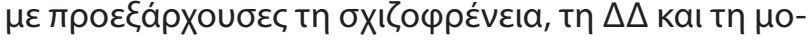
vото入ıкń катá $\theta \lambda ı \psi \eta .^{12}$

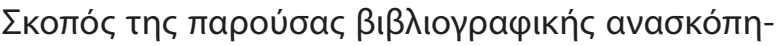

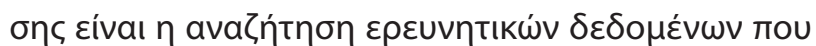

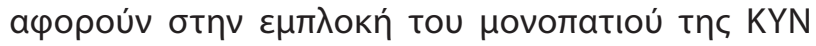

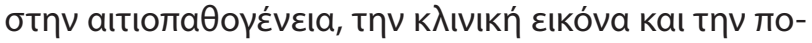

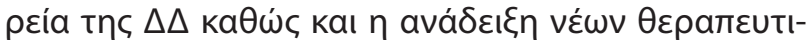

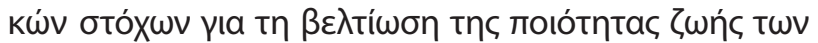

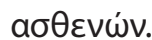

\section{Méசoঠos}

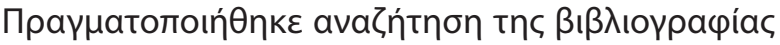

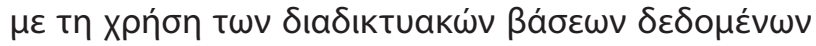

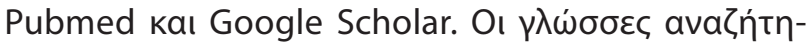

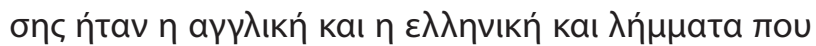
avaそெти́Өெкаv ńtav: "bipolar disorder", "depression", "mania", "tryptophan", "kynurenine pathway", "TRYCAT", "cognitive dysfunction", "sleep disorder", "neuroimmunology", "neuroinflammation".

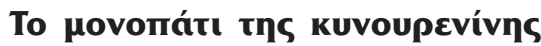

H TRP عívaı a

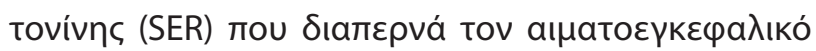

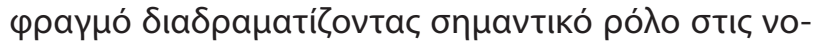

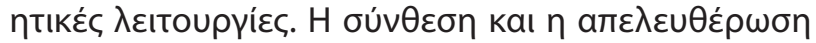

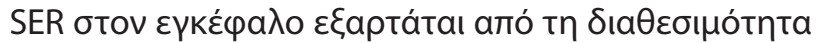

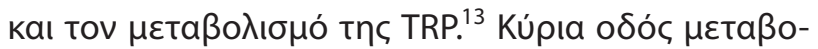

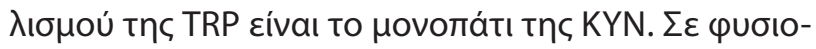

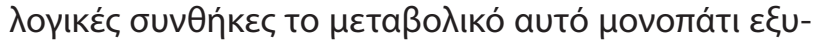

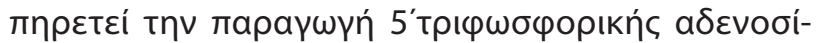

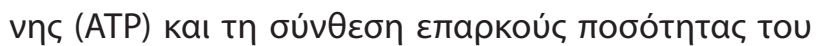

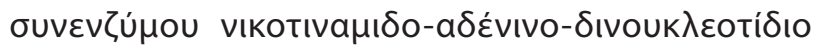

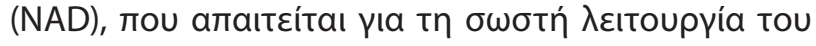

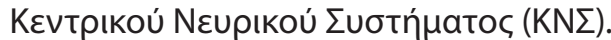

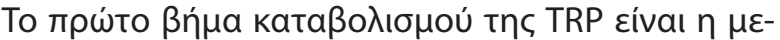

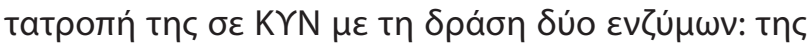




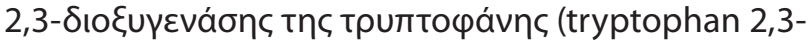

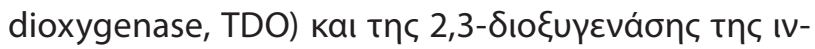

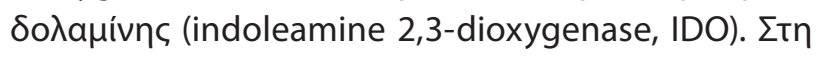

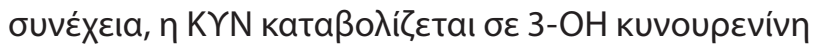

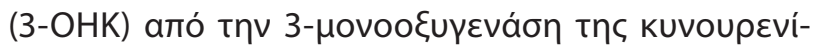

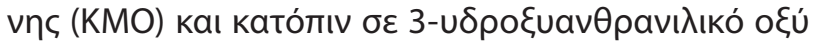

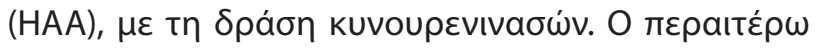

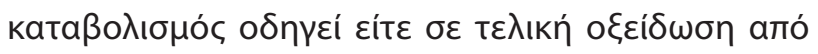

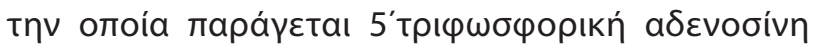

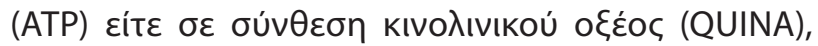

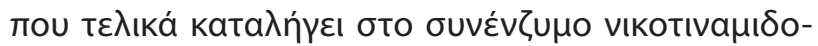

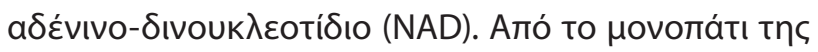

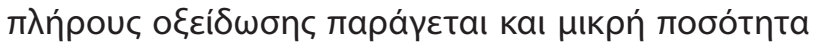

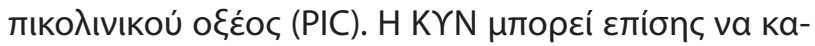

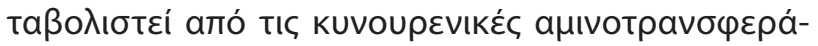

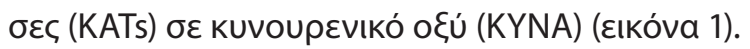

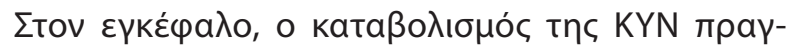

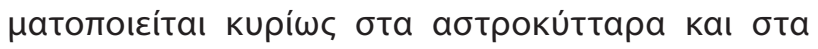

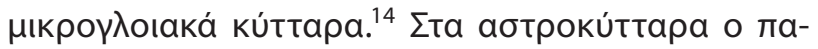

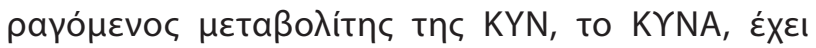

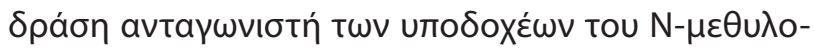

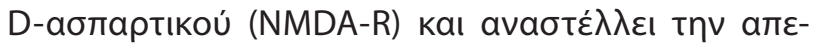

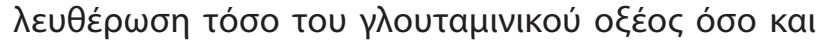

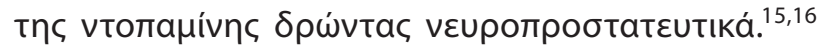

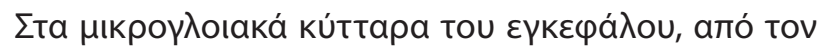

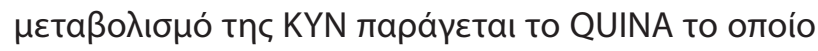

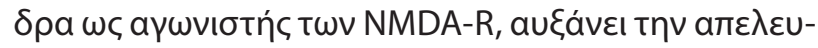

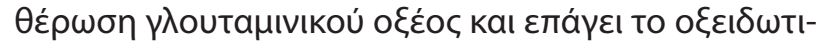

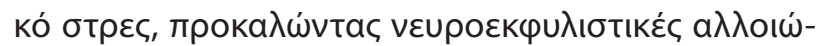

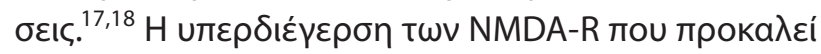

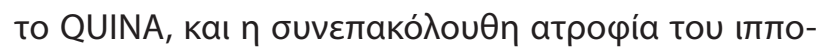

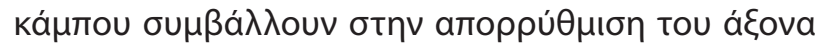

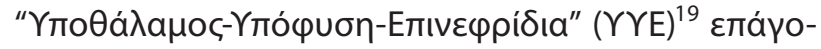

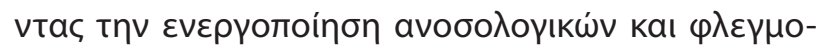

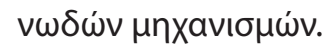

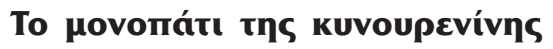

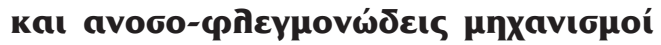

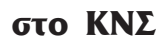

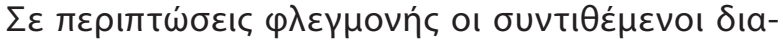

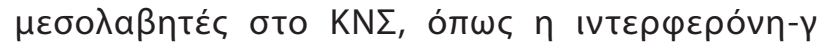

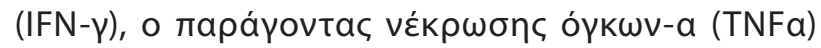

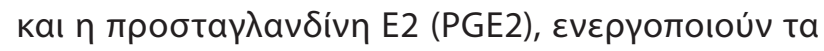

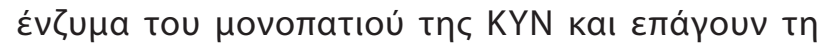

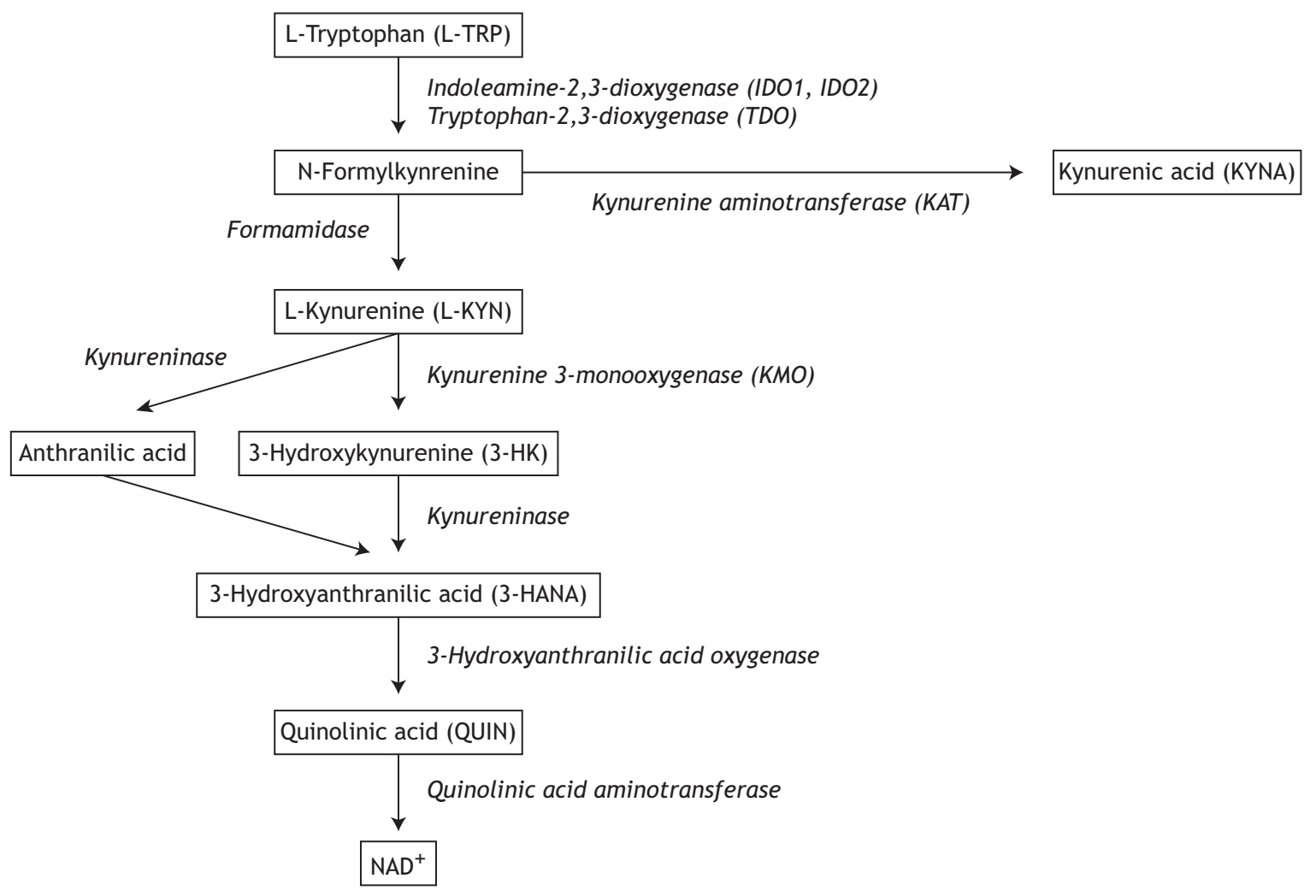

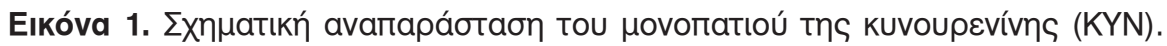




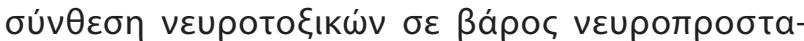

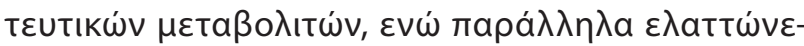

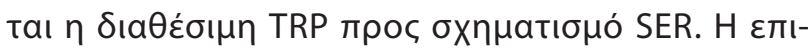

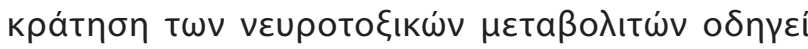

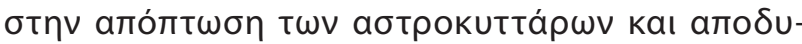

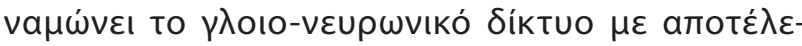

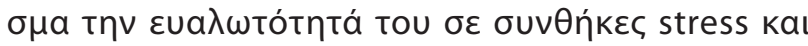

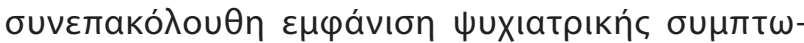

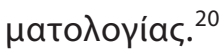

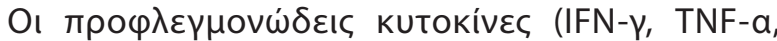

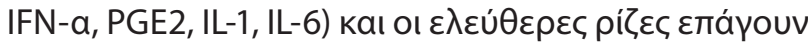

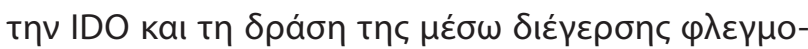

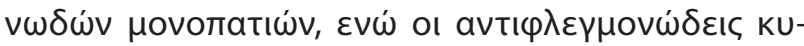

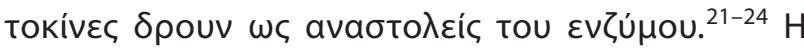

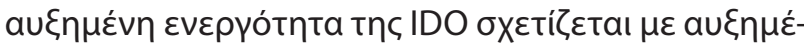

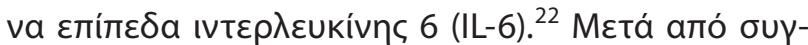

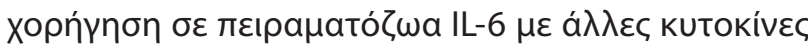

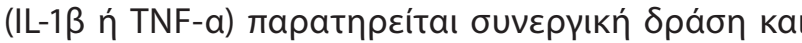

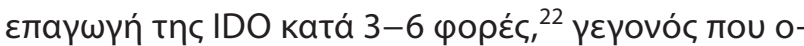

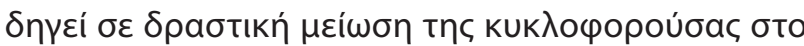

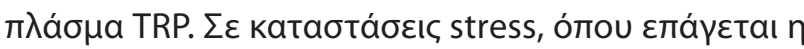

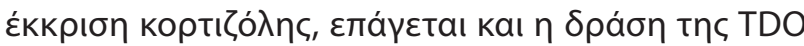

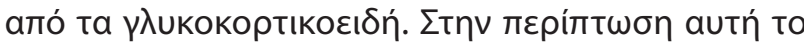

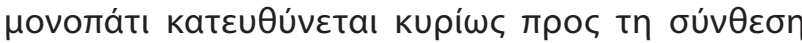

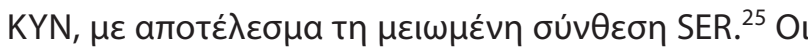

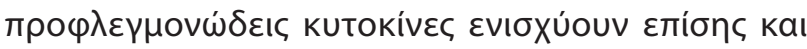

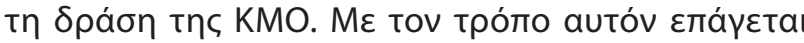

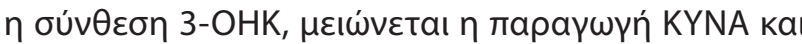

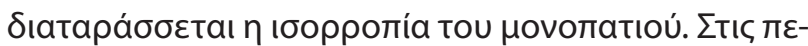

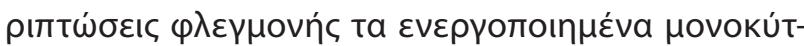

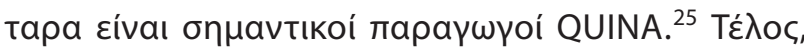

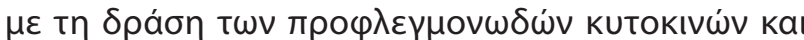

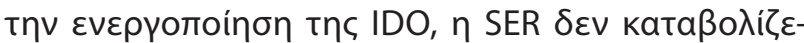

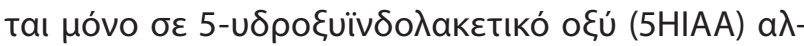

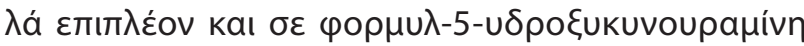

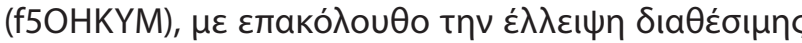

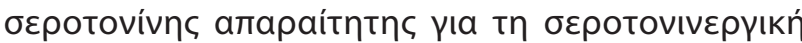
veupodıаßißaon.

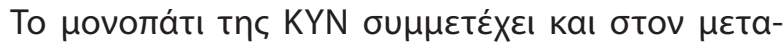

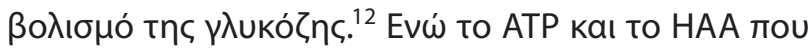

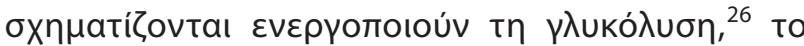

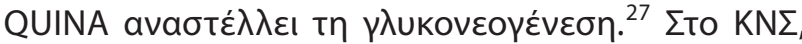

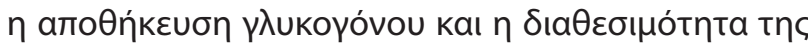

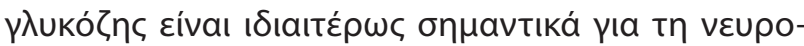

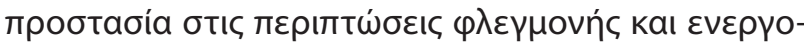

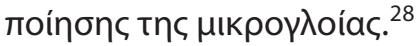

\section{To Hovomáti tns Kuvoupevívns

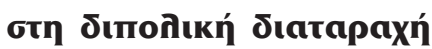

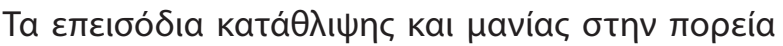

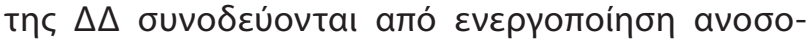

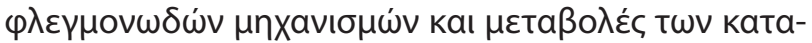

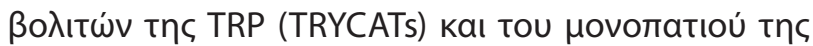

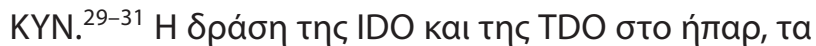

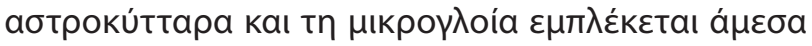

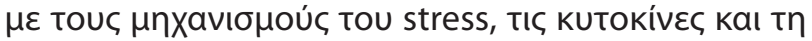

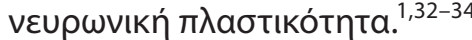

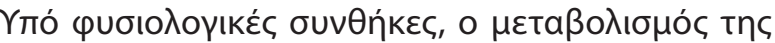

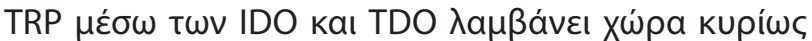

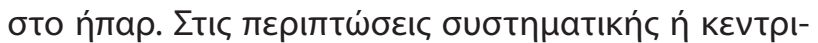

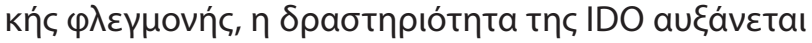

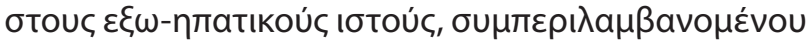

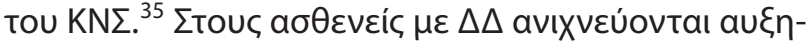

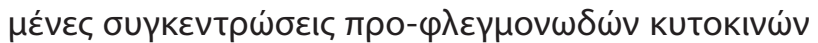

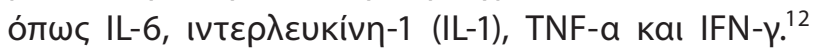

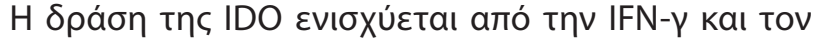

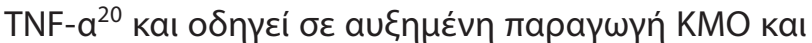

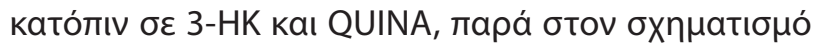

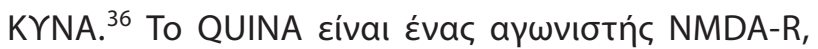

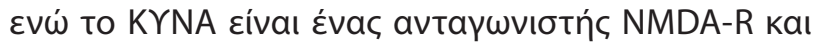

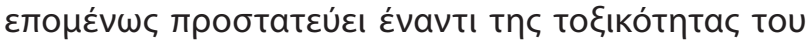

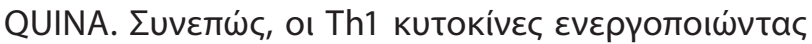

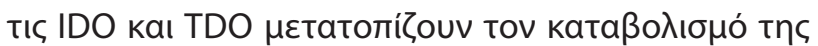

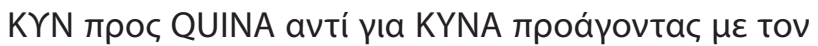

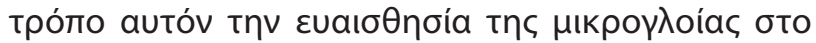

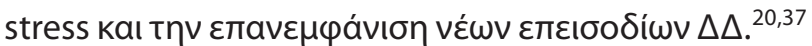

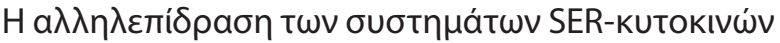

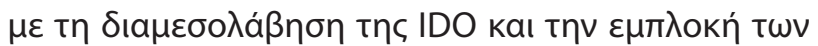

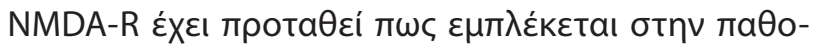

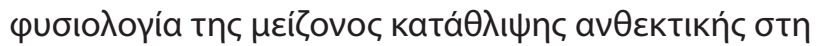

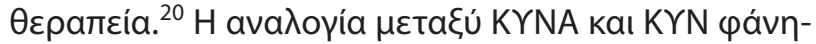

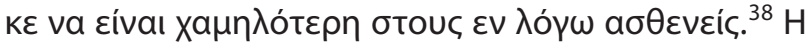

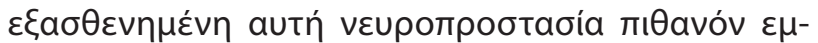

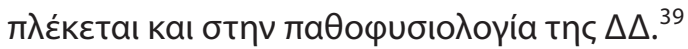

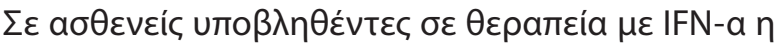

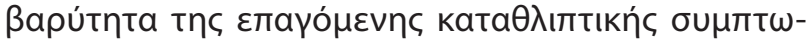

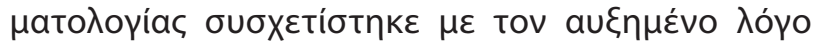

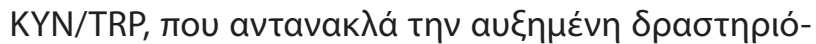

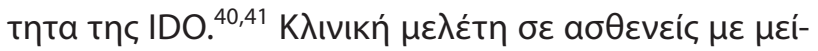

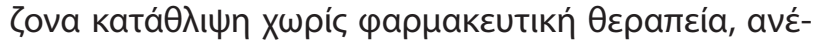

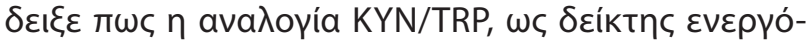

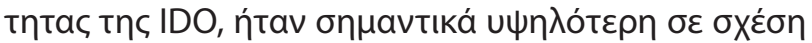




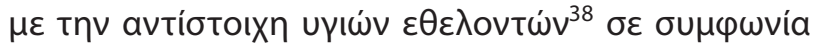

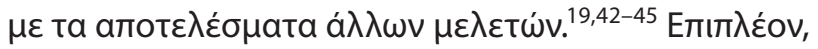

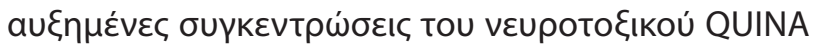

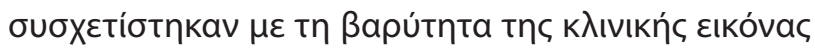

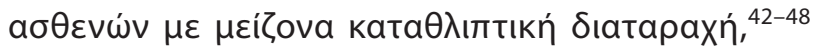

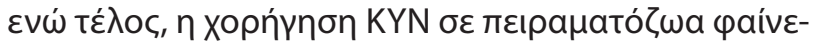

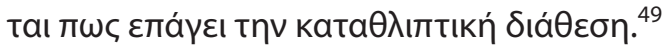

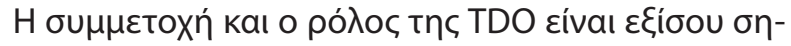

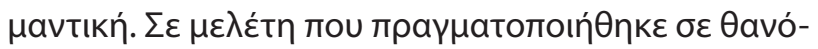

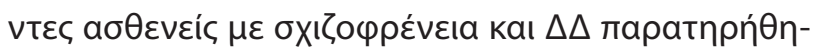

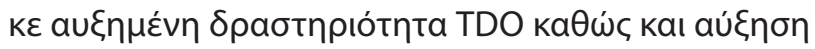

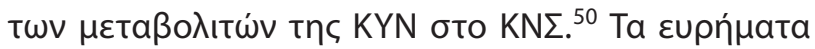

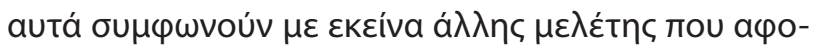

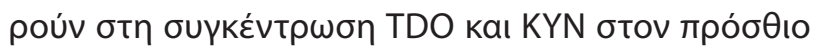

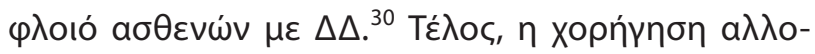

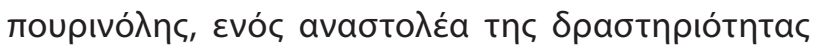

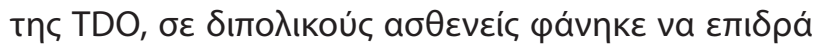
$\theta \varepsilon \tau$ Iкá $\beta \varepsilon \lambda \tau$ t

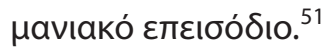

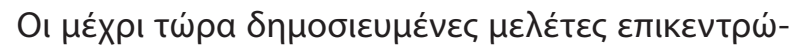

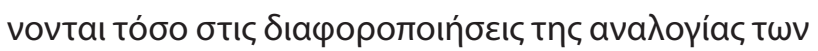

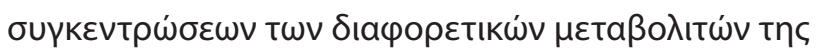

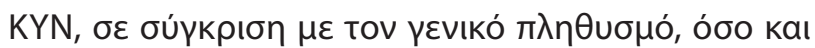

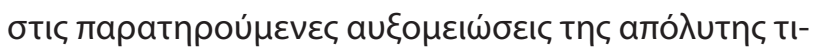

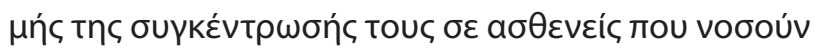

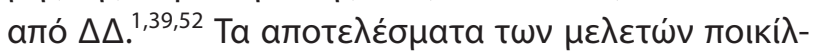

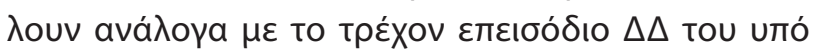
$\mu \varepsilon \lambda \varepsilon \dot{\tau} \eta \eta \lambda \eta \eta \cup u \sigma \mu u ́$.

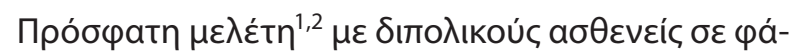

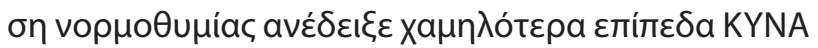

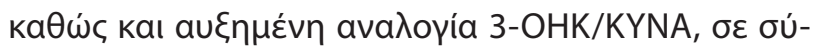

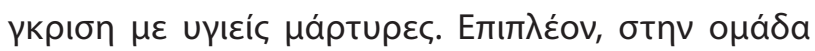

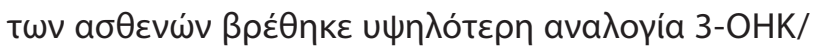

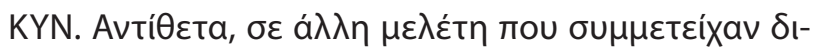

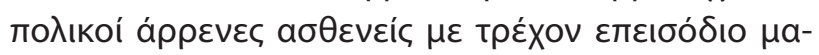

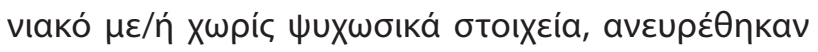

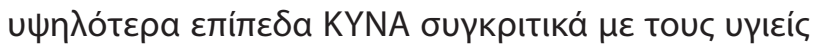

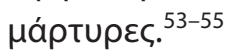

Oı Reininghaus et al ${ }^{52}$ ava

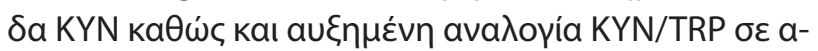

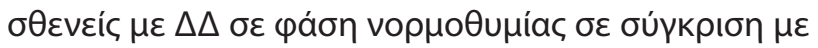

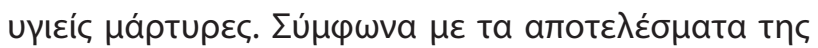

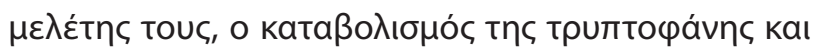

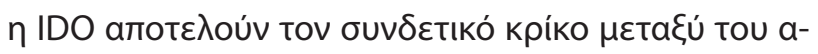

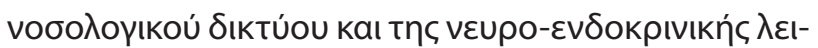

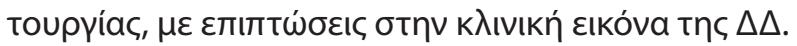

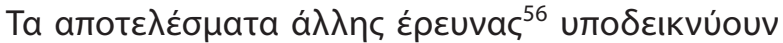

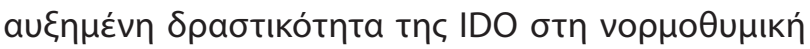

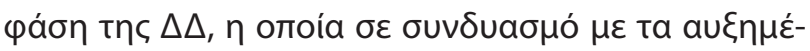

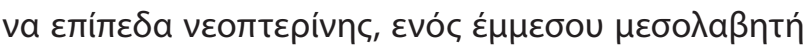

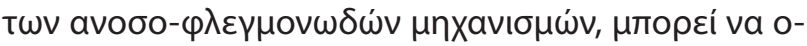

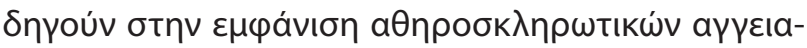

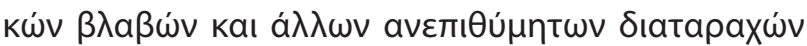

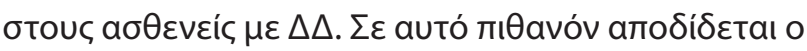

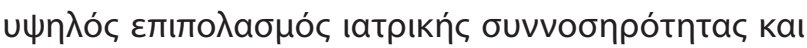

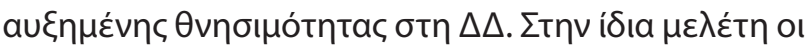

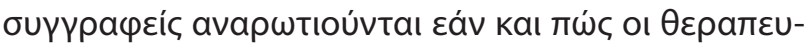

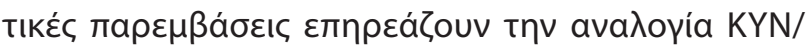

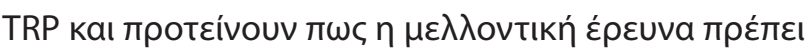

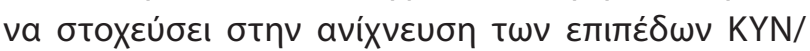

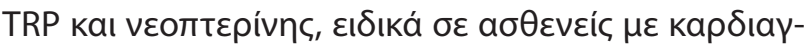

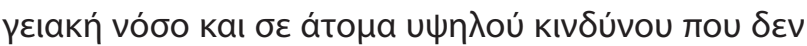

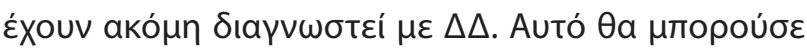

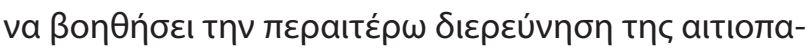

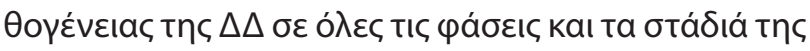

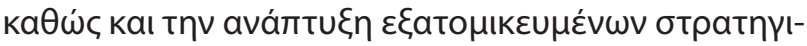

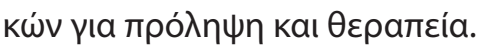

Oı Johansson et $a^{57} \varepsilon \rho \varepsilon u v \omega ́ v t a c$ tov pódo $\tau \omega v$

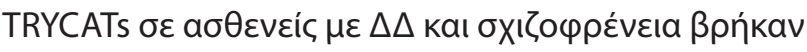

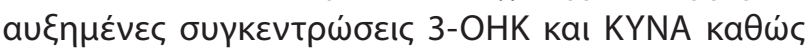

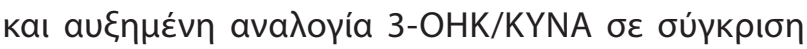

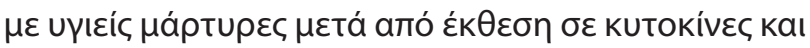

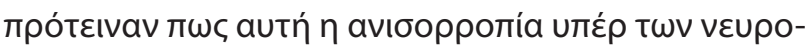

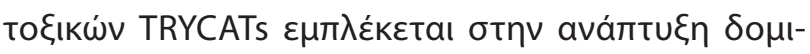

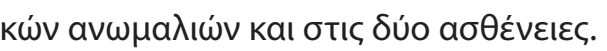

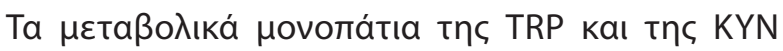

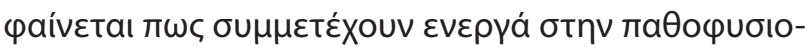

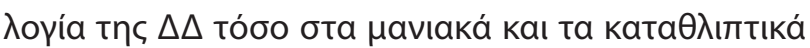

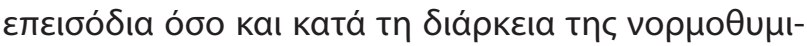

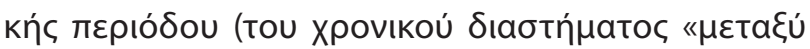

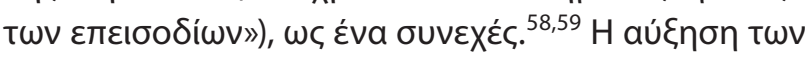

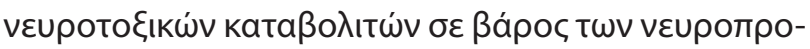

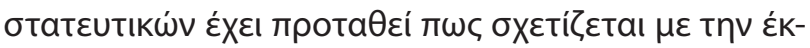

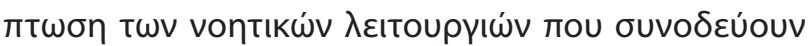

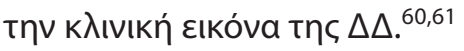

\section{Kuvoupevívn Kal vontukés הeıtToupyícs

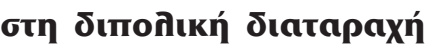

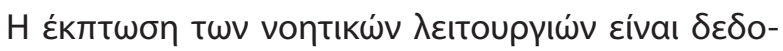

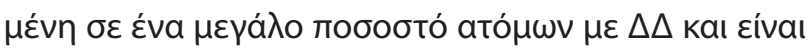

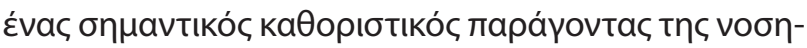

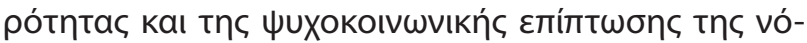




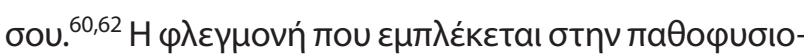

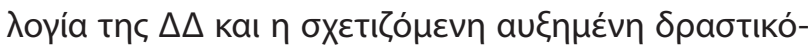

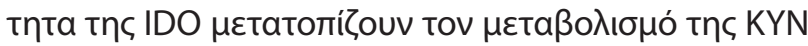

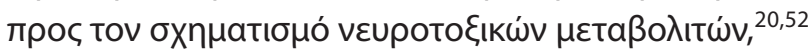

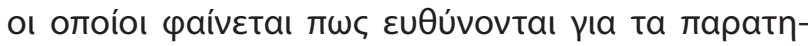

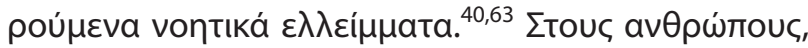

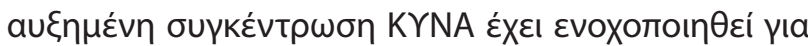

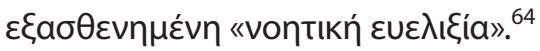

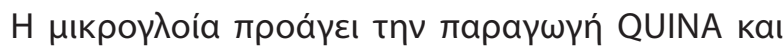

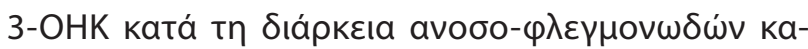

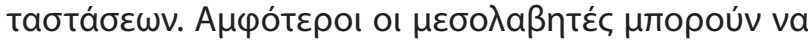

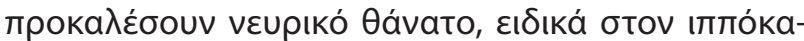

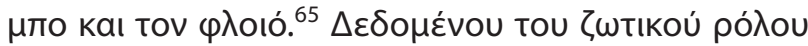

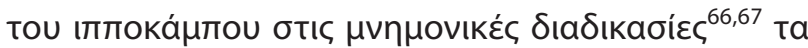

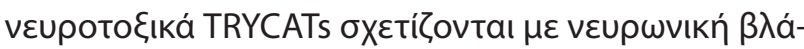

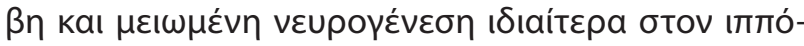

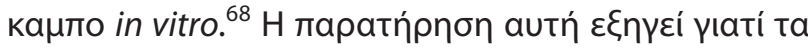

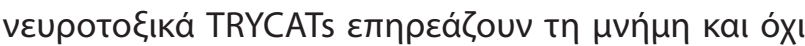

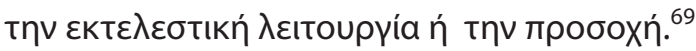

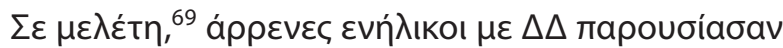

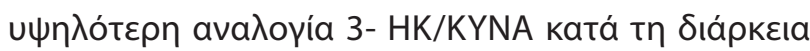

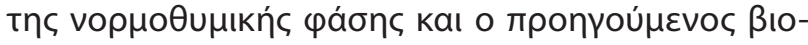

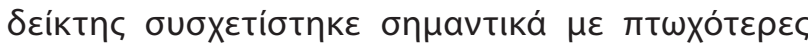

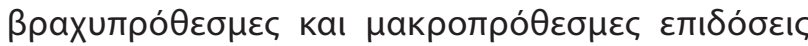

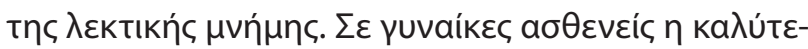

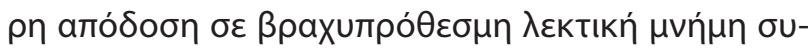

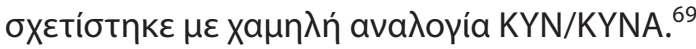

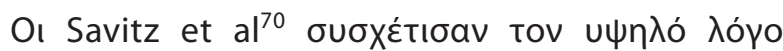

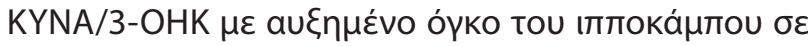

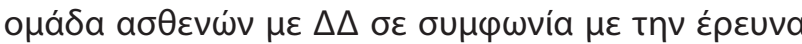

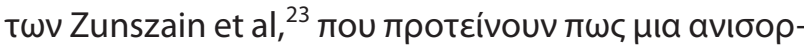

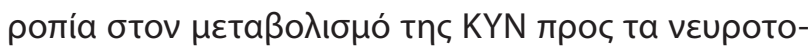

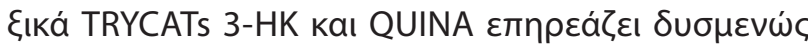

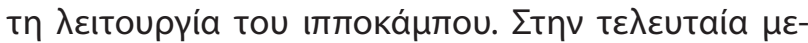

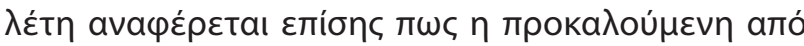

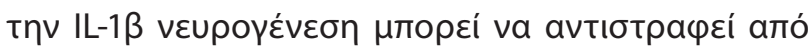

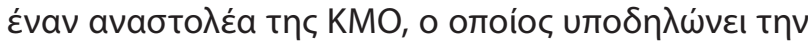

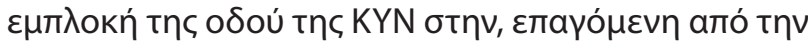

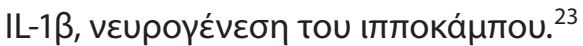

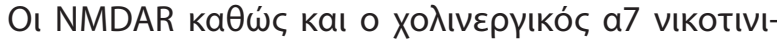

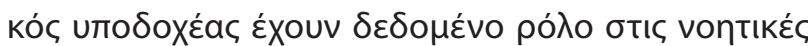

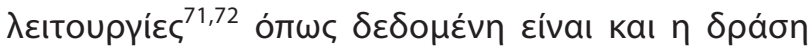

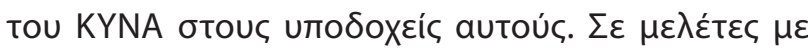

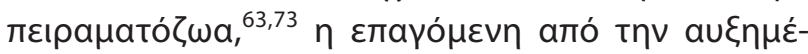

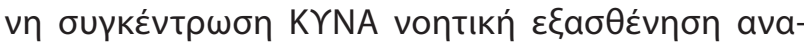
бтра́

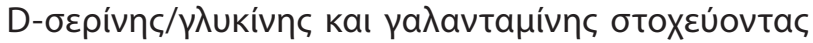

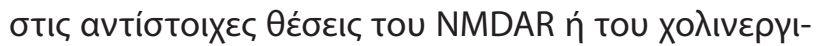

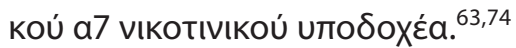

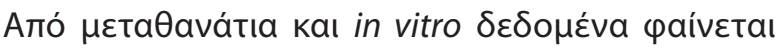

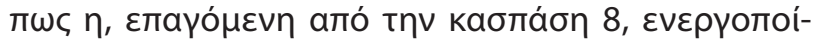

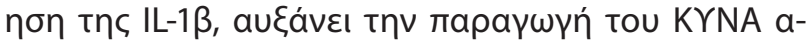

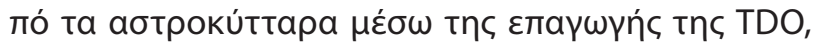

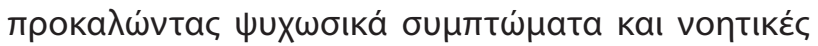

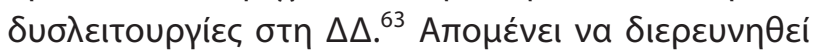

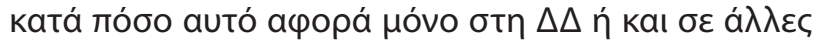

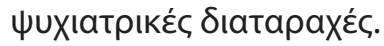

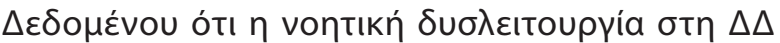

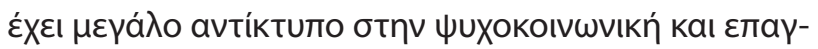

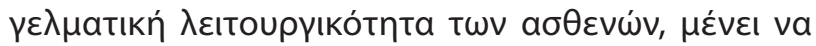

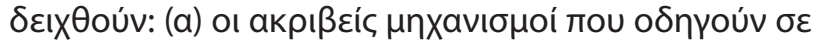

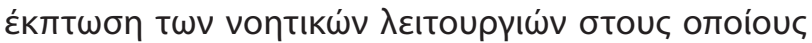

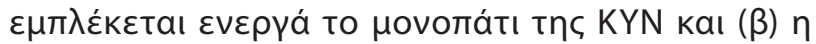

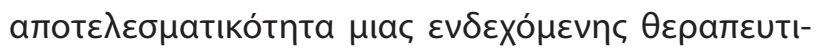

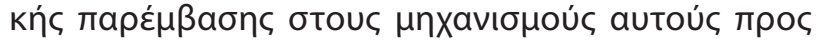

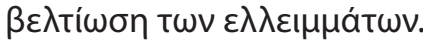

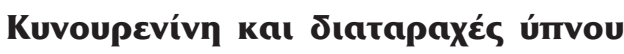

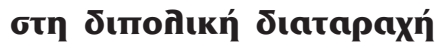

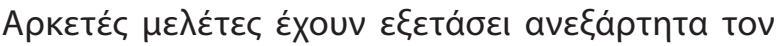

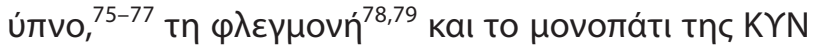

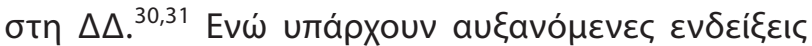

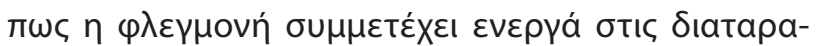

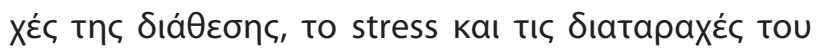

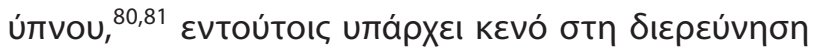

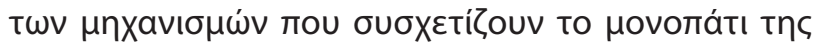

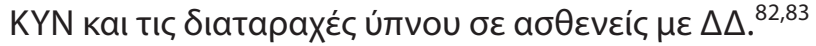

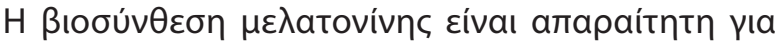

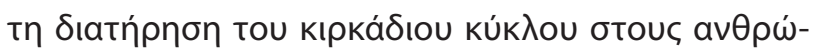

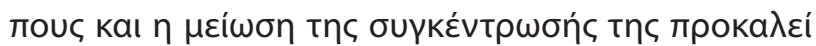

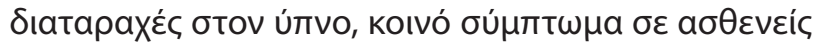

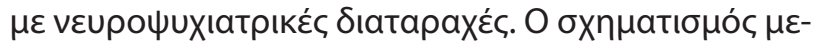

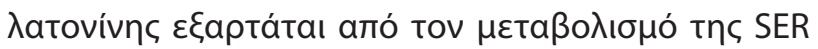

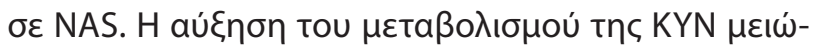

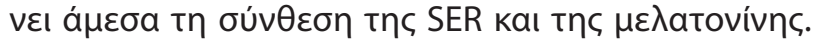

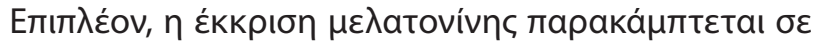

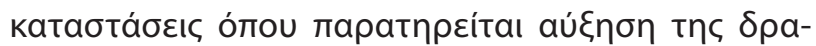

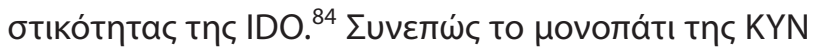

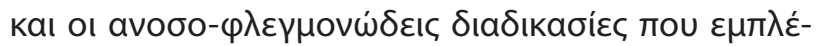

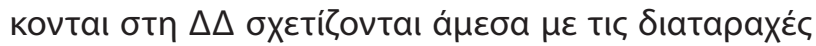

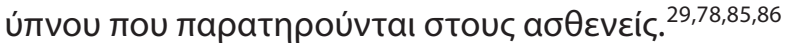




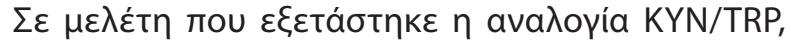

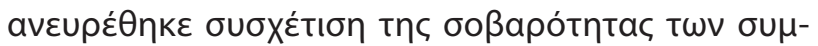

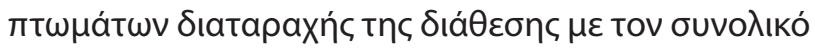

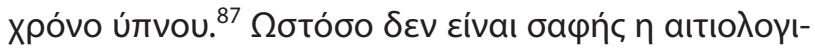

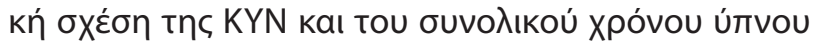

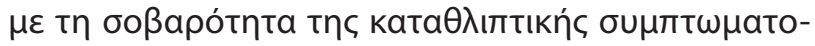

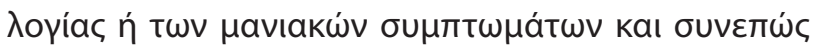

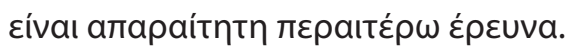

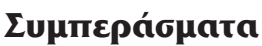

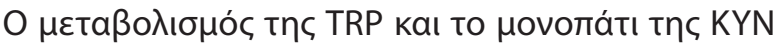

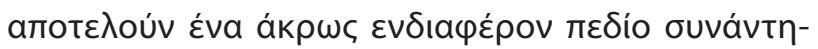

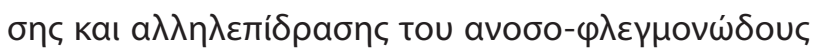

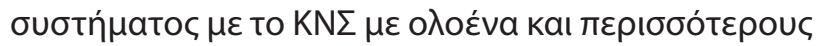

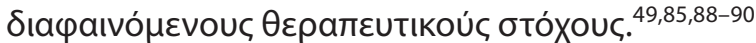

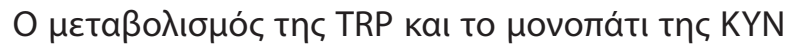

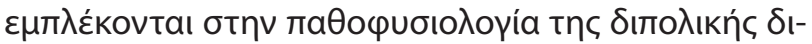

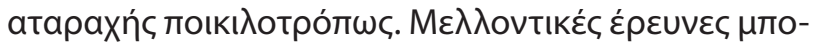

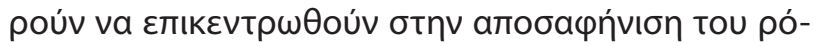

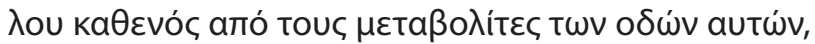

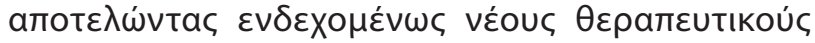

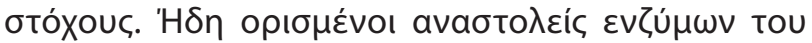

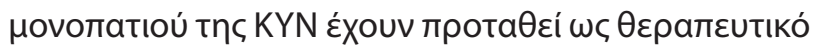

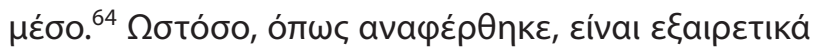

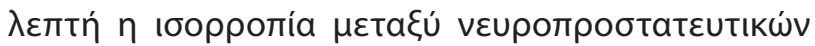

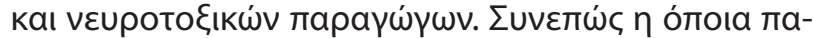

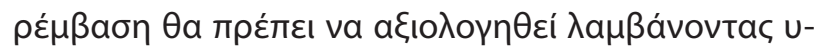

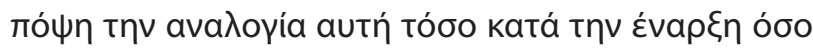

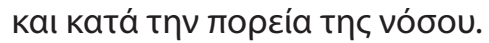

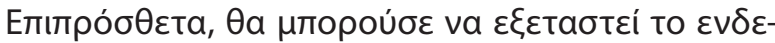

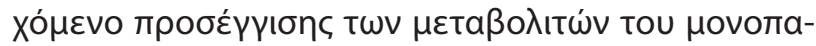

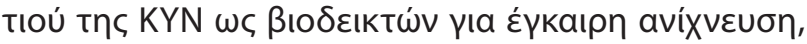

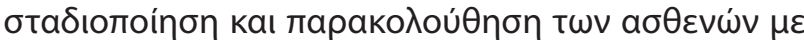

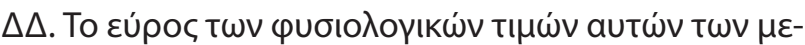

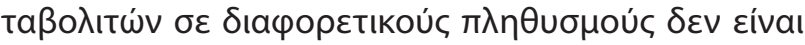

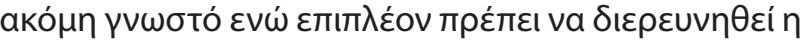

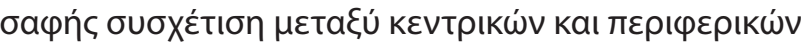

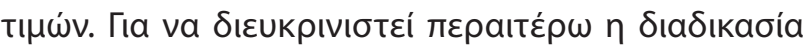

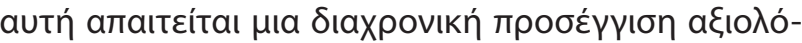

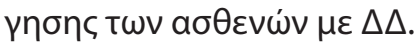

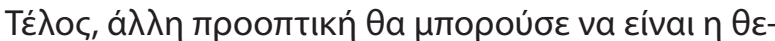

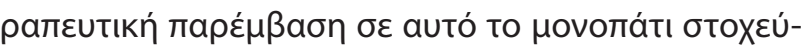

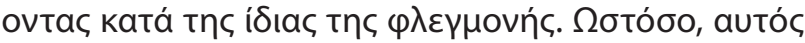

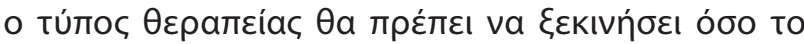

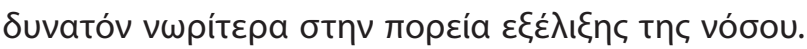

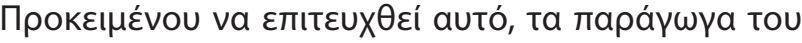

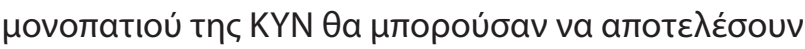

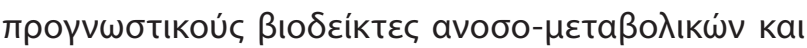

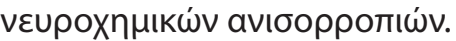

\title{
Kynurenine pathway in bipolar disorder
}

\author{
Ger. Konstantinou, ${ }^{1}$ C.C. Papageorgiou, ${ }^{2}$ E. Angelopoulos ${ }^{2}$ \\ 'Department of Psychiatry, 251 Hellenic Airforce V.A. General Hospital, \\ ${ }^{2} 1$ st Department of Psychiatry, Medical School, National and Kapodistrian University of Athens, \\ Eginition Hospital, Athens, Greece
}

Psychiatriki 2018, 29:338-348

Bipolar disorder (BD) is a chronic psychiatric illness which, among other things, is characterized by cerebral dysfunctions, cognitive disorders and sleep disturbances. The neurobiological basis of these processes remains unclear. In recent years, studies have focused on the role of immune-inflammatory mechanisms induced by the tryptophan metabolism pathway (TRP) and the kynurenine pathway (KYN). Emerging data correlates TRP and KYN metabolites with BD's pathophysiology and course. The purpose of this review is to search the available data on the involvement of KYN's pathway in the pathophysiology, the clinical presentation and the course of $\mathrm{BD}$. A systematic literature review was conducted using 
web-based search engines provided by PubMed (for Medline database) and Google Scholar. The search languages were English and Greek and the entries Key phrases used for the research were: bipolar disorder, depression, mania, tryptophan, kynurenine pathway, cognitive dysfunction, sleep disorder, neuroimmunology, neuroinflammation manuscripts written or published in English and Greek language. The $\mathrm{KYN}$ pathway is actively involved in the pathophysiology of $\mathrm{BD}$. The increase in neurotoxic weight of the neuroprotective derivatives of the pathway is associated with cognitive impairment that accompany the clinical presentation of the disease. In addition, some of these metabolites are also suspected of sleep disorders in BD. Further studies are needed to investigate the mechanisms involved. The KYN pathway is a highly interesting field of encounter and interaction of the immune inflammatory system with the CNS, both involved in the pathophysiology of $\mathrm{BD}$ in a variety of ways. Future research can focus on clarifying the role of the metabolites of this pathway, potentially highlighting new therapeutic goals. Additionally, consideration could be given to approaching the metabolites of the KYN pathway as biomarkers for early detection, staging and monitoring of BD patients.

Key words: Bipolar disorder, tryptophan, kynurenine, cognitive impairment, sleep disorders, serotonin.

\section{BıBהtoypacpía}

1. Birner A, Platzer M, Bengesser SA, Dalkner N, Fellendorf FT, Queissner R et al. Increased breakdown of kynurenine towards its neurotoxic branch in bipolar disorder. PLOS ONE 2017, 12: e0172699, doi: 10.1371/journal.pone.0172699

2. Anderson G, Jacob A, Bellivier F, Geoffroy PA. Bipolar Disorder: The Role of the Kynurenine and Melatonergic Pathways. Curr Pharm Des 2016, 22:987-1-1012. doi: 10.2174/1381612822666 151214105314

3. Stone TW. Neuropharmacology of quinolinic and kynurenic acids. Pharmacol Rev 1993, 45:309-379

4. Gin H. Neurobehavioral dysfunction in uremia. Kidney Int Suppl 1975, 2:217-221

5. Hegedus Z, Frank H, Steinman T, Nayak U. Dialysis of plasma soluble lipofucsins in patients with end-stage renal failure. Arch Int Physiol Biochim Biophys 1999, 99:355-363, doi: 10.3109/13813459109146950

6. Moe S, Sprague S. Uremic encephalophaty. Clin Nephrol 1994, 42:251-256

7. Topczewska-Bruns J, Tankiewicz A, Pawlak D, Buczko W. Behavioral changes in the course of chronic renal insufficiency in rats. Pol J Pharamcol 2001, 53:263-269

8. Holmes EW, Kahn SE. Tryptophan distribution and metabolism in experimental chronic renal insufficiency. Exp Mol Pathol 1987, 46:89-101, doi: 10.1016/0014-4800(87)90033-5

9. Holmes EW, Russell PM, Kinzler GJ, Reckard CR, Flanigan RC, Thompson KD et al. Oxidative tryptophan metabolism in renal allograft recipients: Increased kynurenine synthesis is associated with inflammation and OKT3 therapy. Cytokine 1992, 4:205-213 doi: 10.1016/1043-4666(92)90057-x

10. Saito K, Heyes MP. Kynurenine pathway enzymes in brain-properties of enzymes and regulation of quinolinic acid synthesis. Adv Exp Med Biol 1996, 398:485-492, doi: 10.1007/978-1-46130381-7_75

11. Kawashima Y, Sanaka T, Sugino N, Takahashi M, Mizoguchi $\mathrm{H}$. Suppressive effect of quinolinic acid and hippuric acid on bone marrow erythroid growth and lymphocyte blast formation in uremia. Adv Exp Med Biol 1987, 223:69-72, doi: 10.1007/9781-4684-5445-1_9

12. Myint AM. Kynurenines: from the perspective of major psychiatric disorders. FEBS Journal 2012, 279:1375-1385. doi: 10.1111/ j.1742-4658.2012.08551.x

13. Russo S, Kema IP, Bosker F, Haavik J, Korf J. Tryptophan as an evolutionarily conserved signal to brain serotonin: molecular evidence and psychiatric implications. The world journal of biological psychiatry: the official journal of the World Federation of Societies of Biological Psychiatry 2009, 10:258-268, doi: 10.1080/15622970701513764

14. Schwarcz R, Bruno JP, Muchowski PJ, Wu HQ. Kynurenines in the mammalian brain: when physiology meets pathology. Nat Rev Neurosci 2012, 13:465-477, doi: 10.1038/nrn3257

15. Borland LM, Michael AC. Voltammetric study of the control of striatal dopamine release by glutamate. J Neurochem 2004, 91:220-229, doi: 10.1111/j.1471-4159.2004.02708.x

16. Sloan SA, Barres BA. Mechanisms of astrocyte development and their contributions to neurodevelopmental disorders. Curr Opin Neurobiol 2014, 27:75-81, doi: 10.1016/j.conb.2014.03.005

17. Schwarcz R, Pellicciari R. Manipulation of brain kynurenines: glial targets, neuronal effects, and clinical opportunities. $J$ Pharmacol Experiment Therapeut 2002, 303:1-10, doi: 10.1124/ jpet.102.034439

18. Aguzzi A, Barres BA, Bennett ML. Microglia: scapegoat, saboteur, or something else? Science 2013, 339:156-161, doi: 10.1126/ science. 1227901

19. Wichers MC, Maes M. The role of indoleamine 2,3-dioxygenase (IDO) in the pathophysiology of interferon-a-induced depression. J Psychiatry Neurosci 2004, 29:11-17

20. Myint AM, Kim YK. Cytokine-serotonin interaction through IDO: a neurodegeneration hypothesis of depression. Med Hypotheses 2003, 61:519-525, doi: 10.1016/s0306-9877(03)00207-x

21. Konsman JP, Parnet P, Dantzer R. Cytokine-induced sickness behaviour: mechanisms and implications. Trends Neurosci 2002, 25:154-159, doi: 10.1016/s0166-2236(00)02088-9 
22. Fujigaki H, Saito K, Fujigaki S, Takemura M, Sudo K, Ishiguro $\mathrm{H}$ et al. The signal transducer and activator of transcription 1 alpha and interferon regulatory factor 1 are not essential for the induction of indoleamine 2,3-dioxygenase by lipopolysaccharide: involvement of p38 mitogenactivated protein kinase and nuclear factor-kappaB pathways, and synergistic effect of several proinflammatory cytokines. J Biochem 2006, 139:655-662, doi: 10.1093/jb/mvj072

23. Zunszain PA, Anacker C, Cattaneo A, Choudhury S, Musaelyan $\mathrm{K}$, Myint $\mathrm{AM}$ et al. Interleukin-1 beta: a new regulator of the kynurenine pathway affecting human hippocampal neurogenesis. Neuropsychopharmacology: Official publication of the American College of Neuropsychopharmacology 2012, 37:939-949, doi: 10.1038/npp.2011.277

24. MacKenzie CR, Worku D, Däubener W. Regulation of IDOmediated bacteriostasis in macrophages: role of antibiotics and anti-inflammatory agents. Advanc Experiment Med Biol 2003, 527:67-76, doi: 10.1007/978-1-4615-0135-0_7

25. Myint AM, Schwarz MJ, Müller N. The role of the kynurenine metabolism in major depression. J Neural Transm 2012, 119: 245-251, doi: 10.1007/s00702-011-0741-3

26. Quagliariello E, Papa S, Saccone C, Alifano A. Effect of 3hydroxyanthranilic acid on the mitochondrial respiratory system. Biochem J 1964, 91:137-146, doi: 10.1042/bj0910137

27. Lardy HA. The role of tryptophan metabolites in regulating gluconeogenesis. Am J Clin Nutr 1971, 2:764-765, doi: 10.1093/ ajcn/24.7.764

28. Yuskaitis CJ, Jope RS. Glycogen synthase kinase-3 regulates microglial migration, inflammation, and inflammation-induced neurotoxicity. Cell Signal 2009, 21:264-273, doi: 10.1016/j.cellsig.2008.10.014

29. Anderson G, Maes M. Bipolar disorder: Role of immune-inflammatory cytokines, oxidative and nitrosative stress and tryptophan catabolites. Curr Psychiatry Rep 2015, 17:8-014-0541-1, doi: 10.1007/s11920-014-0541-1

30. Miller CL, Llenos IC, Dulay JR, Weis S. Upregulation of the initiating step of the kynurenine pathway in postmortem anterior cingulate cortex from individuals with schizophrenia and bipolar disorder. Brain Res 2006, 1073:25-37, doi: 10.1016/j. brainres.2005.12.056

31. Clark S, Pocivavsek A, Nicholson J, Notarangelo F, Langenberg $\mathrm{P}$, MacMahon $\mathrm{R}$ et al. Reduced kynurenine pathway metabolism and cytokine expression in the prefrontal cortex of depressed individuals. J Psychiatry Neurosci 2016, 41:368-394, doi: 10.1503/jpn. 150226

32. Anderson G, Maes M. Metabolic syndrome, alzheimer disease, schizophrenia, and depression: Role for leptin, melatonin, kynurenine pathways, and neuropeptides. In: Faroqui TFA (ed) Metabolic Syndome and neurolgical disorders. Wiley, 2013: 235-248, doi:10.1002/9781118395318.ch13

33. Watkins CC, Sawa A, Pomper MG. Glia and immune cell signaling in bipolar disorder: Insights from neuropharmacology and molecular imaging to clinical application. Translation Psychiatr 2014, 4:e350, doi: 10.1038/tp.2013.119

34. Ascoli BM, Géa LP, Colombo R, Barbé-Tuana FM, Kapczinski F, Rosa AR. The role of macrophage polarization on bipolar disorder: Identifying new therapeutic targets. Austr N Zeal J Psychiatr 2016, 50:618-630, doi: 10.1177/0004867416642846
35. Heyes MP, Saito K, Major EO, Milstien S, Markey SP, Vickers $\mathrm{JH}$. A mechanism of quinolinic acid formation by brain in inflammatory neurological disease. Attenuation of synthesis from L-tryptophan by 6-chlorotryptophan and 4-chloro-3- hydroxyanthranilate. Brain: A Journal of Neurology 1993, 116:1425-1450, doi: $10.1093 /$ brain/116.6.1425

36. Munn DH, Shafizadeh E, Attwood JT, Bpndarev I, Pashine A, Mellor AL. Inhibition of T cell proliferation by macrophage tryptophan catabolism. J Experiment Med 1999,189:1363-1372, doi: 10.1084/jem.189.9.1363

37. Dantzer R, O'Connor JC, Freund GG, Johnson RW, Kelley KW. From inflammation to sickness and depression: When the immune system subjugates the brain. Nat Rev Neurosci 2008, 9:46-56, doi: $10.1038 / \mathrm{nrn} 2297$

38. Myint AM, Kim YK, Verkerk R, Scharpe S, Steinbusch $H$, Leonard B. Kynurenine pathway in major depression: evidence of impaired neuroprotection. J Affect Disord 2007, 98:143-151, doi: $10.1016 /$ j.jad.2006.07.013

39. Myint AM, Kim YK, Verkerk R, Park SH, Scharpé S, Steinbusch HW et al. Tryptophan breakdown pathway in bipolar mania. $J$ Affect Disord 2007, 102:65-72, doi: 10.1016/j.jad.2006.12.008

40. Raison CL, Dantzer R, Kelley KW, Lawson MA, Woolwine BJ, Vogt $G$ et al. CSF concentrations of brain tryptophan and kynurenines during immune stimulation with IFN-alpha: relationship to CNS immune responses and depression. Molecul Psychiatr 2010, 15:393-403, doi: 10.1038/mp.2009.116

41. Wichers MC, Koek GH, Robaeys G, Verkerk R, Scharpe S, Maes M. IDO and interferon-alpha-induced depressive symptoms: a shift in hypothesis from tryptophan depletion to neurotoxicity. Molecul Psychiatr 2005, 10:538-544, doi: 10.1038/sj.mp.4001600

42. Gabbay V, Klein RG, Katz Y, Mendoza S, Guttman LE, Alonso $\mathrm{CM}$ et al. The possible role of the kynurenine pathway in adolescent depression with melancholic features. J Child Psychol Psychiatr Allied Disciplin 2010, 51:935-943, doi: 10.1111/j.14697610.2010.02245.x

43. Kim H, Chen L, Lim G, Sung B, Wang S, McCabe MF et al. Brain indoleamine 2,3-dioxygenase contributes to the comorbidity of pain and depression. J Clin Investigat 2012, 122:2940-2954, doi: 10.1172/jci61884

44. Maes M, Rief W. Diagnostic classifications in depression and somatization should include biomarkers, such as disorders in the tryptophan catabolite (TRYCAT) pathway. Psychiatr Research 2012, 196:243-249, doi: 10.1016/j.psychres.2011.09.029

45. Maes M, Verkerk R, Bonaccorso S, Ombelet W, Bosmans E, Scharpe S. Depressive and anxiety symptoms in the early puerperium are related to increased degradation of tryptophan into kynurenine, a phenomenon which is related to immune activation. Life Sci 2002, 71:1837-1848, doi: 10.1016/s0024-3205 (02)01853-2

46. Chiarugi A, Calvani M, Meli E, Traggiai E, Moroni F. Synthesis and release of neurotoxic kynurenine metabolites by human monocytederived macrophages. J Neuroimmunol 2001, 120:190-198, doi: 10.1016/s0165-5728(01)00418-0

47. Steiner J, Walter M, Gos T, Guillemin GJ, Bernstein HG, Sarnyai $Z$ et al. Severe depression is associated with increased microglial quinolinic acid in subregions of the anterior cingulate gyrus: evidence for an immunemodulated glutamatergic neu- 
rotransmission? J Neuroinflammat 2011, 8:94, doi: 10.1016/j. npbr.2012.02.043

48. Müller N, Schwarz MJ. The immune-mediated alteration of serotonin and glutamate: towards an integrated view of depression. Molecul Psychiatr 2007, 12:988-1000, doi: 10.1038/sj.mp. 4002006

49. O'Connor JC, Lawson MA, Andre C, Moreau M, Lestage J, Castanon N. Lipopolyssacharide-induced depressive-like behaviour is mediated by indoleamine 2,3-dioxygenase activation in mice. Mol Psychiatr 2008, 14:511-522, doi: 10.1038/ sj.mp.4002148

50. Miller CL, Llenos IC, Cwik M, Walkup J, Weis S. Alterations in kynurenine precursor and product levels in schizophrenia and bipolar disorder. Neurochemistr Intern 2008, 52:1297-1303, doi: 10.1016/j.neuint.2008.01.013

51. Jahangard L, Soroush S, Haghighi M, Ghaleiha A, Bajoghli H, Holsboer-Trachsler $\mathrm{E}$ et al. In a double-blind, randomized and placebo-controlled trial, adjuvant allopurinol improved symptoms of mania in in-patients suffering from bipolar disorder. European Neuropsychopharmacology: The Journal of the European College of Neuropsychopharmacology 2014, 24:1210-1221, doi: 10.1016/j.euroneuro.2014.05.013

52. Reininghaus EZ, Mclntyre RS, Reininghaus B, Geisler S, Bengesser SA, Lackner $\mathrm{N}$ et al. Tryptophan breakdown is increased in euthymic overweight individuals with bipolar disorder: A preliminary report. Bipol Disord 2014, 16:432-440, doi: 10.1111/bdi.12166

53. Olsson SK, Samuelsson M, Saetre P, Lindstrom L, Jonsson EG, Nordin $C$ et al. Elevated levels of kynurenic acid in the cerebrospinal fluid of patients with bipolar disorder. J Psychiatry Neurosci 2010, 35:195-199, doi: 10.1503/jpn.090180

54. Lavebratt C, Olsson S, Backlund L, Frisén L, Sellgren C, Priebe $\mathrm{L}$ et al. The KMO allele encoding Arg452 is associated with psychotic features in bipolar disorder type 1, and with increased CSF KYNA level and reduced KMO expression. Mol Psychiatr 2013, 19:334-341, doi: 10.1038/mp.2013.11

55. Olsson SK, Sellgren C, Engberg G, Landen M, Erhardt S. Cerebrospinal fluid kynurenic acid is associated with manic and psychotic features in patients with bipolar I disorder. Bipol Disord 2012, 14:719-726, doi: 10.1111/bdi.12009

56. Dalkner N, Platzer M, Bengesser SA, Birner A, Fellendorf FT, Queissner $\mathrm{R}$ et al. The role of tryptophan metabolism and food craving in the relationship between obesity and bipolar disorder. Clin Nutr 2017, doi: 10.1016/j.clnu.2017.06.024

57. Johansson AS, Owe-Larsson B, Asp L, Kocki T, Adler M, Hetta J et al. Activation of kynurenine pathway in ex vivo fibroblasts from patients with bipolar disorder or schizophrenia: Cytokine challenge increases production of 3-hydroxykynurenine. J Psychiatr Res 2013, 47:1815-1823, doi: 10.1016/j.jpsychires.2013.08.008

58. Kim YK, Jung HG, Myint AM, Kim H, Park SH. Imbalance between pro-inflammatory and anti-inflammatory cytokines in bipolar disorder. J Affect Disord 2007, 104:91-95, doi: 10.1016/j. jad.2007.02.018

59. Langan C, McDonald C. Neurobiological trait abnormalities in bipolar disorder. Mol Psychiatr 2009, 14:833-846, doi: 10.1038/ mp.2009.39

60. Robinson LJ, Ferrier IN. Evolution of cognitive impairment in bipolar disorder: A systematic review of cross-sectional evi- dence. Bipol Disord 2006, 8:103-116, doi: 10.1111/j.13995618.2006.00277.x

61. Lackner N, Bengesser SA, Birner A, Painold A, Fellendorf FT, Platzer $\mathrm{M}$ et al. Abdominal obesity is associated with impaired cognitive function in euthymic bipolar individuals. World $\mathrm{J}$ Biol Psychiatr 2015, 1-12, doi: 10.3109/15622975.2015.1046917

62. Arts B, Jabben N, Krabbendam L, van Os J. Meta-analyses of cognitive functioning in euthymic bipolar patients and their firstdegree relatives. Psychol Med 2007, 38:771-785, doi: 10.1017/ s0033291707001675

63. Sellgren CM, Kege ME, Bergen SE, Ekman CJ, Olsson S, Larsson $\mathrm{M}$ et al. A genome-wide association study of kynurenic acid in cerebrospinal fluid: implications for psychosis and cognitive impairment in bipolar disorder. Molecul Psychiatr 2015, 21:13421350, doi: 10.1038/mp.2015.186

64. Stone TW, Forrest CM. Darlington LG. Kynurenine pathway inhibition as a therapeutic strategy for neuroprotection. FEBS $J$ 2012, 279:1386-1397, doi: 10.1111/j.1742-4658.2012.08487.x

65. Chiarugi A, Meli E, Moroni F. Similarities and differences in the neuronal death processes activated by $3 \mathrm{OH}$-kynurenine and quinolinic acid. $J$ Neurochemistr 2001, 77:1310-1318, doi: 10.1046/j.1471-4159.2001.00335.x

66. Cao B, Bauer IE, Sharma AN, Mwangi B, Frazier T, Lavagnino $L$ et al. Reduced hippocampus volume and memory performance in bipolar disorder patients carrying the BDNF val66met met allele. J Affect Disord 2016, 198:198-205, doi: 10.1016/j. jad.2016.03.044

67. Keenan PA, Ezzat WH, Ginsburg K, Moore GJ. Prefrontal cortex as the sight of estrogen's effect on cognition. Psychoneuroendocrinology 2001, 26:577-590, doi: 10.1016/s03064530(01)00013-0

68. Monnerie H, Shashidhara S, Le Roux PD. Effect of excess extracellular glutamate on dendrite growth from cerebral cortical neurons at 3 days in vitro: involvement of NMDA receptors. J Neurosci Res 2003, 74:688-744, doi: 10.1002/jnr.10797

69. Platzer M, Dalkner N, Fellendorf FT, Birner A, Bengesser SA, Queissner R et al. Tryptophan breakdown and cognition in bipolar disorder. Psychoneuroendocrinology 2017, 81:144-151, doi: 10.1016/j.clnu.2017.06.024.

70. Savitz J, Dantzer R, Wurfel BE, Victor TA, Ford BN, Bodurkaet J et al. Neuroprotective kynurenine metabolite indices are abnormally reduced and positively associated with hippocampal and amygdalar volume in bipolar disorder. Psychoneuroendocrinology 2015, 52:200-211, doi: 10.1016/j.psyneuen.2014.11.015

71. Alexander KS, Wu HQ, Schwarcz R, Bruno JP. Acute elevations of brain kynurenic acid impair cognitive flexibility: normalization by the alpha7 positive modulator galantamine. Psychopharmacology 2012, 220:627-637, doi: 10.1007/s00213-011-2539-2

72. Labrie V, Lipina T, Roder JC. Mice with reduced NMDA receptor glycine affinity model some of the negative and cognitive symptoms of schizophrenia. Psychopharmacology 2008, 200:217-230, doi: 10.1007/s00213-008-1196-6

73. Nikiforuk A, Kos T, Rafa D, Behl B, Bespalov A, Popik P. Blockade of glycine transporter 1 by SSR-504734 promotes cognitive flexibility in glycine/NMDA receptor-dependent manner. Neuropharmacology 2011, 61:262-267, doi: 10.1016/j.neuropharm.2011.04.010 
74. Hoekstra R, Fekkes D, Loonen AJ, Pepplinkhuizen L, Tuinier S, Verhoeven WM. Bipolar mania and plasma aminoacids: increased levels of glycine. Eur Neuropsychopharmacol 2006, 16: 71-77, doi: 10.1016/j.euroneuro.2005.06.003

75. Murray G, Harvey A. Circadian rhythms and sleep in bipolar disorder. Bipol Disord 2010, 12:459-472, doi: 10.1111/j.13995618.2010.00843.x

76. Harvey AG, Soehner AM, Kaplan KA, Hein K, Lee J, Kanady J et al. Treating insomnia improves mood state, sleep, and functioning in bipolar disorder: a pilot randomized controlled trial. $J$ Consult Clin Psychol 2015, 83:564, doi: 10.1037/a0038655

77. Kanady JC, Soehnera AM, Harvey AG. A retrospective examination of sleep disturbance across the course of bipolar disorder. J Sleep Disord Ther 2015, 4:193, doi: 10.4172/2167-0277. 1000193

78. Berk M, Kapczinski F, Andreazza A, Dean OM, Giorlando F, Maes $\mathrm{M}$ et al. Pathways underlying neuro- progression in bipolar disorder: focus on inflammation, oxidative stress and neurotrophic factors. Neurosci Biobehav Rev 2011, 35:804-817, doi: 10.1016/j. neubiorev.2010.10.001

79. Goldstein BI, Kemp DE, Soczynska JK, Mclntyre RS. Inflammation and the phenomenology, pathophysiology, comorbidity, and treatment of bipolar disorder: a systematic review of the literature. J Clin Psychiatr 2009, 70:1078-1090, doi: 10.4088/jcp.08r04505

80. Fernandez-Mendoza J, Vgontzas AN. Insomnia and its impact on physical and mental health. Curr Psychiatry Rep 2013, 15:1-8, doi: 10.1007/s11920-013-0418-8

81. Gaines J, Vgontzas AN, Fernandez-Mendoza J, Kritikou I, Basta M, Bixler EO. Gender differences in the association of sleep apnea and inflammation. Brain Behav Immun 2015, 47:211-217, doi: 10.1016/j.bbi.2014.11.013

82. Manji HK, Quiroz JA, Payne JL, Singh A, Lopes BP, Viegas JS et al. The underlying neurobiology of bipolar disorder. Wrld Psychiatr 2003, 2:136
83. Mukherjee D, Krishnamurthy VB, Millett CE, Reider A, Can A, Groer $\mathrm{M}$ et al. Total sleep time and kynurenine metabolism associated with mood symptom severity in bipolar disorder. Bipol Disord 2017, 20:27-34, doi: 10.1111/bdi.12529

84. Oxenkrug GF. Serotonin-kynurenine hypothesis of depression: Historical overview and recent developments. Curr Drug Targ 2013, 14:514-521, doi: 10.2174/1389450111314050002

85. Etain B, Dumaine A, Bellivier F, Pagan C, Francelle L, GoubranBotros $\mathrm{H}$ et al. Genetic and functional abnormalities of the melatonin biosynthesis pathway in patients with bipolar disorder. Hum Molecul Genet 2012, 21:4030-4037, doi: 10.1093/hmg/dds227

86. Harvey AG, Schmidt DA, Scarnü A, Semler CN, Goodwin GM. Sleep-related functioning in euthymic patients with bipolar disorder, patients with insomnia, and subjects without sleep problems. Am J Psychiatry 2005, 162:50-57, doi: 10.1176/appi.ajp.162.1.50

87. Widner B, Laich A, Sperner-Unterweger B, Ledochowski M, Fuchs D. Neopterin production, tryptophan degradation, and mental depression - what is the link? Brain Behav Immun 2002, 16:590-595, doi: 10.1016/s0889-1591(02)00006-5

88. Stone TW. Kynurenic acid antagonists and kynurenine pathway inhibitors. Expert Opin Investing Drugs 2001, 10:633-645, doi: 10.1517/13543784.10.4.633

89. Müller N, Myint AM, Schwarz MJ. Inflammatory Biomarkers and Depression. Neurotox Res 2011, 19:308-318, doi: 10.1007/978-1

90. Boufidou F, Nikolaou C. Anti-inflammatory medication as adjunctive antidepressive treatment. Psychiatriki 2016, 27:106-17, doi: 10.22365/jpsych.2016.272.106

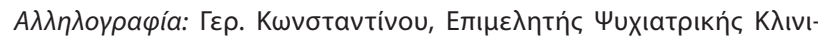

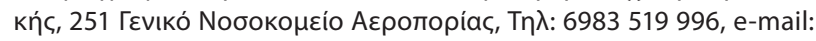
gerkonst@gmail.com 